\title{
Higher-order Frobenius-Euler and poly-Bernoulli mixed-type polynomials
}

\section{Dae San Kim ${ }^{1}$ and Taekyun $\mathrm{Kim}^{2 *}$}

${ }^{\text {*Correspondence: tkkim@kw.ac.kr }}$
${ }^{2}$ Department of Mathematics,
Kwangwoon University, Seoul,
$139-701$, Republic of Korea
Full list of author information is
available at the end of the article

available at the end of the article

\begin{abstract}
In this paper, we consider higher-order Frobenius-Euler polynomials, associated with poly-Bernoulli polynomials, which are derived from polylogarithmic function. These polynomials are called higher-order Frobenius-Euler and poly-Bernoulli mixed-type polynomials. The purpose of this paper is to give various identities of those polynomials arising from umbral calculus.
\end{abstract}

\section{Introduction}

For $\lambda \in \mathbb{C}$ with $\lambda \neq 1$, the Frobenius-Euler polynomials of order $\alpha(\alpha \in \mathbb{R})$ are defined by the generating function to be

$$
\left(\frac{1-\lambda}{e^{t}-\lambda}\right)^{\alpha} e^{x t}=\sum_{n=0}^{\infty} H_{n}^{(\alpha)}(x \mid \lambda) \frac{t^{n}}{n !} \quad(\text { see }[1-5])
$$

When $x=0, H_{n}^{(\alpha)}(\lambda)=H_{n}^{(\alpha)}(0 \mid \lambda)$ are called the Frobenius-Euler numbers of order $\alpha$. As is well known, the Bernoulli polynomials of order $\alpha$ are defined by the generating function to be

$$
\left(\frac{t}{e^{t}-1}\right)^{\alpha} e^{x t}=\sum_{n=0}^{\infty} \mathbb{B}_{n}^{(\alpha)}(x) \frac{t^{n}}{n !} \quad(\text { see }[6-8]) .
$$

When $x=0, \mathbb{B}_{n}^{(\alpha)}=\mathbb{B}_{n}^{(\alpha)}(x)$ is called the $n$th Bernoulli number of order $\alpha$. In the special case, $\alpha=1, \mathbb{B}_{n}^{(1)}(x)=B_{n}(x)$ is called the $n$th Bernoulli polynomial. When $x=0, B_{n}=B_{n}(0)$ is called the $n$th ordinary Bernoulli number. Finally, we recall that the Euler polynomials of order $\alpha$ are given by

$$
\left(\frac{2}{e^{t}+1}\right)^{\alpha} e^{x t}=\sum_{n=0}^{\infty} E_{n}^{(\alpha)}(x) \frac{t^{n}}{n !} \quad(\text { see }[9-13])
$$

When $x=0, E_{n}^{(\alpha)}=E_{n}^{(\alpha)}(0)$ is called the $n$th Euler number of order $\alpha$. In the special case, $\alpha=1, E_{n}^{(1)}(x)=E_{n}(x)$ is called the $n$th ordinary Euler polynomial. The classical polylogarithmic function $L i_{k}(x)$ is defined by

$$
L i_{k}(x)=\sum_{n=1}^{\infty} \frac{x^{n}}{n^{k}} \quad(k \in \mathbb{Z})(\text { see }[7]) .
$$

\section{Springer}

( 2013 Kim and Kim; licensee Springer. This is an Open Access article distributed under the terms of the Creative Commons Attribution License (http://creativecommons.org/licenses/by/2.0), which permits unrestricted use, distribution, and reproduction in any medium, provided the original work is properly cited. 
As is known, poly-Bernoulli polynomials are defined by the generating function to be

$$
\frac{L i_{k}\left(1-e^{-t}\right)}{1-e^{-t}} e^{x t}=\sum_{n=0}^{\infty} B_{n}^{(k)}(x) \frac{t^{n}}{n !} \quad(c f .[7])
$$

Let $\mathbb{C}$ be the complex number field, and let $\mathcal{F}$ be the set of all formal power series in the variable $t$ over $\mathbb{C}$ with

$$
\mathcal{F}=\left\{f(t)=\sum_{k=0}^{\infty} \frac{a_{k}}{k !} t^{k} \mid a_{k} \in \mathbb{C}\right\} .
$$

Now, we use the notation $\mathbb{P}=\mathbb{C}[x]$. In this paper, $\mathbb{P}^{*}$ will be denoted by the vector space of all linear functionals on $\mathbb{P}$. Let us assume that $\langle L \mid p(x)\rangle$ be the action of the linear functional $L$ on the polynomial $p(x)$, and we remind that the vector space operations on $\mathbb{P}^{*}$ are defined by $\langle L+M \mid p(x)\rangle=\langle L \mid p(x)\rangle+\langle M \mid p(x)\rangle,\langle c L \mid p(x)\rangle=c\langle L \mid p(x)\rangle$, where $c$ is a complex constant in $\mathbb{C}$. The formal power series

$$
f(t)=\sum_{k=0}^{\infty} \frac{a_{k}}{k !} t^{k} \in \mathcal{F}
$$

defines a linear functional on $\mathbb{P}$ by setting

$$
\left.\left\langle f(t) \mid x^{n}\right\rangle=a_{n}, \quad \text { for all } n \geq 0 \text { (see }[14,15]\right) .
$$

From (1.7) and (1.8), we note that

$$
\left\langle t^{k} \mid x^{n}\right\rangle=n ! \delta_{n, k} \quad(\text { see }[14,15]),
$$

where $\delta_{n, k}$ is the Kronecker symbol.

Let us consider $f_{L}(t)=\sum_{k=0}^{\infty} \frac{\left\langle L \mid x^{n}\right\rangle}{k !} t^{k}$. Then we see that $\left\langle f_{L}(t) \mid x^{n}\right\rangle=\left\langle L \mid x^{n}\right\rangle$, and so $L=f_{L}(t)$ as linear functionals. The map $L \mapsto f_{L}(t)$ is a vector space isomorphism from $\mathbb{P}^{*}$ onto $\mathcal{F}$. Henceforth, $\mathcal{F}$ will denote both the algebra of formal power series in $t$ and the vector space of all linear functionals on $\mathbb{P}$, and so an element $f(t)$ of $\mathcal{F}$ will be thought of as both a formal power series and a linear functional (see [14]). We shall call $\mathcal{F}$ the umbral algebra. The umbral calculus is the study of umbral algebra. The order $o(f(t))$ of a nonzero power series $f(t)$ is the smallest integer $k$, for which the coefficient of $t^{k}$ does not vanish. A series $f(t)$ is called a delta series if $o(f(t))=1$, and an invertible series if $o(f(t))=0$. Let $f(t), g(t) \in \mathcal{F}$. Then we have

$$
\langle f(t) g(t) \mid p(x)\rangle=\langle f(t) \mid g(t) p(x)\rangle=\langle g(t) \mid f(t) p(x)\rangle \quad \text { (see [14]). }
$$

For $f(t), g(t) \in \mathcal{F}$ with $o(f(t))=1, o(g(t))=0$, there exists a unique sequence $S_{n}(x)$ $\left(\operatorname{deg} S_{n}(x)=n\right)$ such that $\left\langle g(t) f(t)^{k} \mid S_{n}(x)\right\rangle=n ! \delta_{n, k}$ for $n, k \geq 0$. The sequence $S_{n}(x)$ is called the Sheffer sequence for $(g(t), f(t))$, which is denoted by $S_{n}(x) \sim(g(t), f(t))$ (see $\left.[14,15]\right)$. Let $f(t) \in \mathcal{F}$ and $p(t) \in \mathbb{P}$. Then we have

$$
f(t)=\sum_{k=0}^{\infty}\left\langle f(t) \mid x^{k}\right\rangle \frac{t^{k}}{k !}, \quad p(x)=\sum_{k=0}^{\infty}\left\langle t^{k}|p(x)| \frac{x^{k}}{k !} .\right.
$$


From (1.11), we note that

$$
p^{(k)}(0)=\left\langle t^{k} \mid p(x)\right\rangle=\left\langle 1 \mid p^{(k)}(x)\right\rangle
$$

By (1.12), we get

$$
t^{k} p(x)=p^{(k)}(x)=\frac{d^{k} p(x)}{d x^{k}} \quad(\text { see }[14,15]) .
$$

From (1.13), we easily derive the following equation

$$
e^{y t} p(x)=p(x+y), \quad\left\langle e^{y t} \mid p(x)\right\rangle=p(y) .
$$

For $p(x) \in \mathbb{P}, f(t) \in \mathcal{F}$, it is known that

$$
\langle f(t) \mid x p(x)\rangle=\left\langle\partial_{t} f(t) \mid p(x)\right\rangle=\left\langle f^{\prime}(t) \mid p(x)\right\rangle \quad(\text { see [14]). }
$$

Let $S_{n}(x) \sim(g(t), f(t))$. Then we have

$$
\frac{1}{g(\bar{f}(x))} e^{y \bar{f}(t)}=\sum_{n=0}^{\infty} S_{n}(y) \frac{t^{n}}{n !} \quad \text { for all } y \in \mathbb{C},
$$

where $\bar{f}(t)$ is the compositional inverse of $f(t)$ with $\bar{f}(f(t))=t$, and

$$
f(t) S_{n}(x)=n S_{n-1}(x) \quad(\text { see }[14,15])
$$

The Stirling number of the second kind is defined by the generating function to be

$$
\left(e^{t}-1\right)^{m}=m ! \sum_{l=m}^{\infty} S_{2}(l, m) \frac{t^{m}}{m !} \quad\left(m \in \mathbb{Z}_{\geq 0}\right) .
$$

For $S_{n}(x) \sim(g(t), t)$, it is well known that

$$
S_{n+1}(x)=\left(x-\frac{g^{\prime}(t)}{g(t)}\right) S_{n}(x) \quad(n \geq 0)(\text { see }[14,15]) .
$$

Let $S_{n}(x) \sim(g(t), f(t)), r_{n}(x) \sim(h(t), l(t))$. Then we have

$$
S_{n}(x)=\sum_{m=0}^{n} C_{n, m} r_{m}(x)
$$

where

$$
C_{n, m}=\frac{1}{m !}\left\langle\frac{h(\bar{f}(t))}{g(\bar{f}(t))} l(\bar{f}(t))^{m} \mid x^{n}\right\rangle \quad(\text { see }[14,15]) .
$$

In this paper, we study higher-order Frobeniuns-Euler polynomials associated with polyBernoulli polynomials, which are called higher-order Frobenius-Euler and poly-Beroulli mixed-type polynomials. The purpose of this paper is to give various identities of those polynomials arising from umbral calculus. 


\section{Higher-order Frobenius-Euler polynomials, associated poly-Bernoulli polynomials}

Let us consider the polynomials $T_{n}^{(r, k)}(x \mid \lambda)$, called higher-order Frobenius-Euler and polyBernoulli mixed-type polynomials, as follows:

$$
\left(\frac{1-\lambda}{e^{t}-\lambda}\right)^{r} \frac{L i_{k}\left(1-e^{-t}\right)}{1-e^{-t}} e^{x t}=\sum_{n=0}^{\infty} T_{n}^{(r, k)}(x \mid \lambda) \frac{t^{n}}{n !}
$$

where $\lambda \in \mathbb{C}$ with $\lambda \neq 1, r, k \in \mathbb{Z}$.

When $x=0, T_{n}^{(r, k)}(\lambda)=T_{n}^{(r, k)}(0 \mid \lambda)$ is called the $n$th higher-order Frobenius-Euler and poly-Bernoulli mixed type number.

From (1.16) and (2.1), we note that

$$
T_{n}^{(r, k)}(x \mid \lambda) \sim\left(g_{r, k}(t)=\left(\frac{e^{t}-\lambda}{1-\lambda}\right)^{r} \frac{1-e^{-t}}{L i_{k}\left(1-e^{-t}\right)}, t\right) .
$$

By (1.17) and (2.2), we get

$$
t T_{n}^{(r, k)}(x \mid \lambda)=n T_{n-1}^{(r, k)}(x \mid \lambda)
$$

From (2.1), we can easily derive the following equation

$$
\begin{aligned}
T_{n}^{(r, k)}(x \mid \lambda) & =\sum_{l=0}^{n}\left(\begin{array}{l}
n \\
l
\end{array}\right) H_{n-l}^{(r)}(\lambda) B_{l}^{(k)}(x) \\
& =\sum_{l=0}^{n}\left(\begin{array}{l}
n \\
l
\end{array}\right) H_{n-l}^{(r)}(x \mid \lambda) B_{l}^{(k)} .
\end{aligned}
$$

By (1.16) and (2.2), we get

$$
T_{n}^{(r, k)}(x \mid \lambda)=\frac{1}{g_{r, k}(t)} x^{n}=\left(\frac{1-\lambda}{e^{t}-\lambda}\right)^{r} \frac{L i_{k}\left(1-e^{-t}\right)}{1-e^{-t}} x^{n} .
$$

In [7], it is known that

$$
\frac{L i_{k}\left(1-e^{-t}\right)}{1-e^{-t}} x^{n}=\sum_{m=0}^{n} \frac{1}{(m+1)^{k}} \sum_{j=0}^{m}(-1)^{j}\left(\begin{array}{c}
m \\
j
\end{array}\right)(x-j)^{n} .
$$

Thus, by (2.5) and (2.6), we get

$$
\begin{aligned}
T_{n}^{(r, k)}(x \mid \lambda) & =\left(\frac{1-\lambda}{e^{t}-\lambda}\right)^{r} \frac{L i_{k}\left(1-e^{-t}\right)}{1-e^{-t}} x^{n} \\
& =\sum_{m=0}^{\infty} \frac{1}{(m+1)^{k}} \sum_{j=0}^{m}(-1)^{j}\left(\begin{array}{c}
m \\
j
\end{array}\right)\left(\frac{1-\lambda}{e^{t}-\lambda}\right)^{r}(x-j)^{n} \\
& =\sum_{m=0}^{n} \frac{1}{(m+1)^{k}} \sum_{j=0}^{m}(-1)^{j}\left(\begin{array}{c}
m \\
j
\end{array}\right) H_{n}^{(r)}(x-j \mid \lambda) .
\end{aligned}
$$


By (1.1), we easily see that

$$
H_{n}^{(r)}(x \mid \lambda)=\sum_{l=0}^{n}\left(\begin{array}{l}
n \\
l
\end{array}\right) H_{n-l}^{(r)}(\lambda) x^{l}
$$

Therefore, by (2.7) and (2.8), we obtain the following theorem.

Theorem 2.1 For $r, k \in \mathbb{Z}, n \geq 0$, we have

$$
\begin{aligned}
T_{n}^{(r, k)}(x \mid \lambda) & =\sum_{m=0}^{n} \frac{1}{(m+1)^{k}} \sum_{j=0}^{m}(-1)^{j}\left(\begin{array}{c}
m \\
j
\end{array}\right) \sum_{l=0}^{n}\left(\begin{array}{c}
n \\
l
\end{array}\right) H_{n-l}^{(r)}(\lambda)(x-j)^{l} \\
& =\sum_{l=0}^{n}\left\{\left(\begin{array}{l}
n \\
l
\end{array}\right) H_{n-l}^{(r)}(\lambda) \sum_{m=0}^{\infty} \frac{1}{(m+1)^{k}} \sum_{j=0}^{m}(-1)^{j}\left(\begin{array}{c}
m \\
j
\end{array}\right)\right\}(x-j)^{l} .
\end{aligned}
$$

In [7], it is known that

$$
\frac{L i_{k}\left(1-e^{-t}\right)}{1-e^{-t}} x^{n}=\sum_{j=0}^{n}\left\{\sum_{m=0}^{n-j} \frac{(-1)^{n-m-j}}{(m+1)^{k}}\left(\begin{array}{l}
n \\
j
\end{array}\right) m ! S_{2}(n-j, m)\right\} x^{j}
$$

By (2.5) and (2.9), we get

$$
\begin{aligned}
T_{n}^{(r, k)}(x \mid \lambda) & =\left(\frac{1-\lambda}{e^{t}-\lambda}\right)^{r} \frac{L i_{k}\left(1-e^{-t}\right)}{1-e^{-t}} x^{n} \\
& =\sum_{j=0}^{n}\left\{\sum_{m=0}^{n-j} \frac{(-1)^{n-m-j}}{(m+1)^{k}}\left(\begin{array}{c}
n \\
j
\end{array}\right) m ! S_{2}(n-j, m)\right\}\left(\frac{1-\lambda}{e^{t}-\lambda}\right)^{r} x^{j} \\
& =\sum_{j=0}^{n}\left\{\sum_{m=0}^{n-j} \frac{(-1)^{n-m-j}}{(m+1)^{k}}\left(\begin{array}{c}
n \\
j
\end{array}\right) m ! S_{2}(n-j, m)\right\} H_{j}^{(r)}(x \mid \lambda) .
\end{aligned}
$$

Therefore, by (2.8) and (2.10), we obtain the following theorem.

Theorem 2.2 For $r, k \in \mathbb{Z}, n \in \mathbb{Z}_{\geq 0}$, we have

$$
T_{n}^{(r, k)}(x \mid \lambda)=\sum_{l=0}^{n}\left\{\sum_{j=l}^{n} \sum_{m=0}^{n-j}(-1)^{n-m-j}\left(\begin{array}{l}
n \\
j
\end{array}\right)\left(\begin{array}{l}
j \\
l
\end{array}\right) \frac{m !}{(m+1)^{k}} H_{j-l}^{(r)}(\lambda) S_{2}(n-j, m)\right\} x^{l} .
$$

From (1.19) and (2.2), we have

$$
T_{n+1}^{(r, k)}(x \mid \lambda)=\left(x-\frac{g_{r, k}^{\prime}(t)}{g_{r, k}(t)}\right) T_{n}^{(r, k)}(x \mid \lambda) .
$$


Now, we note that

$$
\begin{aligned}
\frac{g_{r, k}^{\prime}(t)}{g_{r, k}(t)} & =\left(\log g_{r, k}(t)\right)^{\prime} \\
& =\left(r \log \left(e^{t}-\lambda\right)-r \log (1-\lambda)+\log \left(1-e^{-t}\right)-\log L i_{k}\left(1-e^{t}\right)\right)^{\prime} \\
& =r+\frac{r \lambda}{e^{t} \lambda}+\left(\frac{t}{e^{t}-1}\right) \frac{L i_{k}\left(1-e^{-t}\right)-L i_{k-1}\left(1-e^{-t}\right)}{t L i_{k}\left(1-e^{-t}\right)}
\end{aligned}
$$

By (2.11) and (2.12), we get

$$
\begin{aligned}
T_{n+1}^{(r, k)}(x \mid \lambda)= & x T_{n}^{(r, k)}(x \mid \lambda)-r T_{n}^{(r, k)}(x \mid \lambda)-\frac{r \lambda}{1-\lambda}\left(\frac{1-\lambda}{e^{t}-\lambda}\right)^{r+1} \frac{L i_{k}\left(1-e^{-t}\right)}{1-e^{-t}} x^{n} \\
& -\left(\frac{1-\lambda}{e^{t}-\lambda}\right)^{r} \frac{L i_{k}\left(1-e^{-t}\right)-L i_{k-1}\left(1-e^{-t}\right)}{t\left(1-e^{-t}\right)}\left(\frac{t}{e^{t}-1}\right) x^{n} \\
= & (x-r) T_{n}^{(r, k)}(x \mid \lambda)-\frac{r \lambda}{1-\lambda} T_{n}^{(r+1, k)}(x \mid \lambda) \\
& -\sum_{l=0}^{n}\left(\begin{array}{l}
n \\
l
\end{array}\right) B_{n-l}\left(\frac{1-\lambda}{e^{t}-\lambda}\right)^{r} \frac{L i_{k}\left(1-e^{-t}\right)-L i_{k-1}\left(1-e^{-t}\right)}{t\left(1-e^{-t}\right)} x^{l} .
\end{aligned}
$$

It is easy to show that

$$
\begin{aligned}
\frac{L i_{k}\left(1-e^{-t}\right)-L i_{k-1}\left(1-e^{-t}\right)}{1-e^{-t}} & =\frac{1}{1-e^{-t}} \sum_{n=1}^{\infty}\left\{\frac{\left(1-e^{-t}\right)^{n}}{n^{k}}-\frac{\left(1-e^{-t}\right)^{n}}{n^{k-1}}\right\} \\
& =\left(\frac{1-e^{-t}}{2^{k}}-\frac{1-e^{-t}}{2^{k-1}}\right)+\cdots \\
& =\left(\frac{1}{2^{k}}-\frac{1}{2^{k-1}}\right) t+\cdots
\end{aligned}
$$

For any delta series $f(t)$, we have

$$
\frac{f(t)}{t} x^{n}=f(t) \frac{1}{n+1} x^{n+1}
$$

Thus, by (2.13), (2.14) and (2.15), we get

$$
\begin{aligned}
T_{n+1}^{(r, k)}(x \mid \lambda)= & (x-r) T_{n}^{(r, k)}(x \mid \lambda)-\frac{r \lambda}{1-\lambda} T_{n}^{(r+1, k)}(x \mid \lambda) \\
& -\sum_{l=0}^{n}\left(\begin{array}{l}
n \\
l
\end{array}\right) B_{n-l} \frac{1}{l+1}\left(\frac{1-\lambda}{e^{t}-\lambda}\right)^{r} \frac{L i_{k}\left(1-e^{-t}\right)-L i_{k-1}\left(1-e^{-t}\right)}{1-e^{-t}} x^{l+1} \\
= & (x-r) T_{n}^{(r, k)}(x \mid \lambda)-\frac{r \lambda}{1-\lambda} T_{n}^{(r+1, k)}(x \mid \lambda) \\
& -\sum_{l=0}^{n} \frac{\left(\begin{array}{l}
n \\
l
\end{array}\right)}{l+1} B_{n-l}\left\{T_{l+1}^{(r, k)}(x \mid \lambda)-T_{l+1}^{(r, k-1)}(x \mid \lambda)\right\} \\
= & (x-r) T_{n}^{(r, k)}(x \mid \lambda)-\frac{r \lambda}{1-\lambda} T_{n}^{(r+1, k)}(x \mid \lambda)
\end{aligned}
$$




$$
\begin{aligned}
& -\frac{1}{n+1} \sum_{l=1}^{n+1}\left(\begin{array}{c}
n+1 \\
l
\end{array}\right) B_{n+1-l}\left\{T_{l}^{(r, k)}(x \mid \lambda)-T_{l}^{(r, k-1)}(x \mid \lambda)\right\} \\
= & (x-r) T_{n}^{(r, k)}(x \mid \lambda)-\frac{r \lambda}{1-\lambda} T_{n}^{(r+1, k)}(x \mid \lambda) \\
& -\frac{1}{n+1} \sum_{l=0}^{n+1}\left(\begin{array}{c}
n+1 \\
l
\end{array}\right) B_{n+1-l}\left\{T_{l}^{(r, k)}(x \mid \lambda)-T_{l}^{(r, k-1)}(x \mid \lambda)\right\} \\
= & (x-r) T_{n}^{(r, k)}(x \mid \lambda)-\frac{r \lambda}{1-\lambda} T_{n}^{(r+1, k)}(x \mid \lambda) \\
& -\frac{1}{n+1} \sum_{l=0}^{n+1}\left(\begin{array}{c}
n+1 \\
l
\end{array}\right) B_{l}\left\{T_{n+1-l}^{(r, k)}(x \mid \lambda)-T_{n+1-l}^{(r, k-1)}(x \mid \lambda)\right\} .
\end{aligned}
$$

Therefore, by (2.16), we obtain the following theorem.

Theorem 2.3 For $r, k \in \mathbb{Z}, n \in \mathbb{Z}_{\geq 0}$, we have

$$
\begin{aligned}
T_{n+1}^{(r, k)}(x \mid \lambda)= & (x-r) T_{n}^{(r, k)}(x \mid \lambda)-\frac{r \lambda}{1-\lambda} T_{n}^{(r+1, k)}(x \mid \lambda) \\
& -\frac{1}{n+1} \sum_{l=0}^{n+1}\left(\begin{array}{c}
n+1 \\
l
\end{array}\right) B_{l}\left\{T_{n+1-l}^{(r, k)}(x \mid \lambda)-T_{n+1-l}^{(r, k-1)}(x \mid \lambda)\right\} .
\end{aligned}
$$

Remark 1 If $r=0$, then we have

$$
\sum_{n=0}^{\infty} B_{n}^{(k)}(x) \frac{t^{n}}{n !}=\frac{L i_{k}\left(1-e^{-t}\right)}{\left(1-e^{-t}\right)} e^{x t}=\sum_{n=0}^{\infty} T_{n}^{(0, k)}(x \mid \lambda) \frac{t^{n}}{n !}
$$

Thus, by (2.17), we get $B_{n}^{(k)}(x)=T_{n}^{(0, k)}(x \mid \lambda)$.

From (2.4), we have

$$
\begin{aligned}
t x T_{n}^{(r, k)}(x \mid \lambda) & =t\left(x \sum_{l=0}^{n}\left(\begin{array}{l}
n \\
l
\end{array}\right) H_{n-l}^{(r)}(\lambda) B_{l}^{(k)}(x)\right) \\
& =\sum_{l=0}^{n}\left(\begin{array}{l}
n \\
l
\end{array}\right) H_{n-l}^{(r)}(\lambda)\left\{l x B_{l-1}^{(k)}(x)+B_{l}^{(k)}(x)\right\} \\
& =n x \sum_{l=0}^{n-1}\left(\begin{array}{c}
n-1 \\
l
\end{array}\right) H_{n-1-l}^{(r)}(\lambda) B_{l}^{(k)}(x)+\sum_{l=0}^{n}\left(\begin{array}{l}
n \\
l
\end{array}\right) H_{n-l}^{(r)}(\lambda) B_{l}^{(k)}(x) \\
& =n x T_{n-1}^{(r, k)}(x \mid \lambda)+T_{n}^{(r, k)}(x \mid \lambda) .
\end{aligned}
$$

Applying $t$ on both sides of Theorem 2.3, we get

$$
\begin{aligned}
(n+1) T_{n}^{(r, k)}(x \mid \lambda) & \\
= & n x T_{n-1}^{(r, k)}(x \mid \lambda)+T_{n}^{(r, k)}(x \mid \lambda)-r n T_{n-1}^{(r, k)}(x \mid \lambda)-\frac{r n \lambda}{1-\lambda} T_{n-1}^{(r+1, k)}(x \mid \lambda) \\
& \quad-\frac{1}{n+1} \sum_{l=0}^{n+1}\left(\begin{array}{c}
n+1 \\
l
\end{array}\right) B_{l}\left\{(n+1-l) T_{n-l}^{(r, k)}(x \mid \lambda)-(n+1-l) T_{n-l}^{(r, k-1)}(x \mid \lambda)\right\} .
\end{aligned}
$$


Thus, by (2.19), we have

$$
\begin{gathered}
(n+1) T_{n}^{(r, k)}(x \mid \lambda)+n\left(r-\frac{1}{2}-x\right) T_{n-1}^{(r, k)}(x \mid \lambda)+\sum_{l=0}^{n-2}\left(\begin{array}{l}
n \\
l
\end{array}\right) B_{n-l} T_{l}^{(r, k)}(x \mid \lambda) \\
=-\frac{r \lambda n}{1-\lambda} T_{n-1}^{(r+1, k)}(x \mid \lambda)+\sum_{l=0}^{n}\left(\begin{array}{l}
n \\
l
\end{array}\right) B_{n-l} T_{l}^{(r, k-1)}(x \mid \lambda) .
\end{gathered}
$$

Therefore, by (2.20), we obtain the following theorem.

Theorem 2.4 For $r, k \in \mathbb{Z}, n \in \mathbb{Z}$ with $n \geq 2$, we have

$$
\begin{gathered}
(n+1) T_{n}^{(r, k)}(x \mid \lambda)+n\left(r-\frac{1}{2}-x\right) T_{n-1}^{(r, k)}(x \mid \lambda)+\sum_{l=0}^{n-2}\left(\begin{array}{l}
n \\
l
\end{array}\right) B_{n-l} T_{l}^{(r, k)}(x \mid \lambda) \\
=-\frac{r \lambda n}{1-\lambda} T_{n-1}^{(r+1, k)}(x \mid \lambda)+\sum_{l=0}^{n}\left(\begin{array}{l}
n \\
l
\end{array}\right) B_{n-l} T_{l}^{(r, k-1)}(x \mid \lambda) .
\end{gathered}
$$

From (1.14) and (2.5), we note that

$$
\begin{aligned}
T_{n}^{(r, k)}(y \mid \lambda) & =\left\langle\left(\frac{1-\lambda}{e^{t}-\lambda}\right)^{r} \frac{L i_{k}\left(1-e^{-t}\right)}{1-e^{-t}} e^{y t} \mid x^{n}\right\rangle \\
& =\left\langle\left(\frac{1-\lambda}{e^{t}-\lambda}\right)^{r} \frac{L i_{k}\left(1-e^{-t}\right)}{1-e^{-t}} e^{y t} \mid x x^{n-1}\right\rangle .
\end{aligned}
$$

By (1.15) and (2.21), we get

$$
\begin{aligned}
T_{n}^{(r, k)}(y \mid \lambda)= & \left\langle\partial_{t}\left(\left(\frac{1-\lambda}{e^{t}-\lambda}\right)^{r} \frac{L i_{k}\left(1-e^{-t}\right)}{1-e^{-t}} e^{y t}\right) \mid x^{n-1}\right\rangle \\
= & \left\langle\left(\partial_{t}\left(\frac{1-\lambda}{e^{t}-\lambda}\right)^{r}\right) \frac{L i_{k}\left(1-e^{-t}\right)}{1-e^{-t}} e^{y t} \mid x^{n-1}\right\rangle \\
& +\left\langle\left(\frac{1-\lambda}{e^{t}-\lambda}\right)^{r}\left(\partial_{t} \frac{L i_{k}\left(1-e^{-t}\right)}{1-e^{-t}}\right) e^{y t} \mid x^{n-1}\right\rangle \\
& +\left\langle\left(\frac{1-\lambda}{e^{t}-\lambda}\right)^{r} \frac{L i_{k}\left(1-e^{-t}\right)}{1-e^{-t}} \partial_{t} e^{y t} \mid x^{n-1}\right\rangle
\end{aligned}
$$

Therefore, by (2.22), we obtain the following theorem.

Theorem 2.5 For $r, k \in \mathbb{Z}, n \geq 1$, we have

$$
\begin{aligned}
T_{n}^{(r, k)}(x \mid \lambda)= & (x-r) T_{n-1}^{(r, k)}(x \mid \lambda)-\frac{r \lambda}{1-\lambda} T_{n-1}^{(r+1, k)}(x \mid \lambda) \\
& +\sum_{l=0}^{n-1}\left\{(-1)^{n-1-l}\left(\begin{array}{c}
n-1 \\
l
\end{array}\right) \sum_{m=0}^{n-1-l}(-1)^{m} \frac{(m+1) !}{(m+2)^{k}} S_{2}(n-1-l, m)\right\} H_{l}^{(r)}(x-1 \mid \lambda) .
\end{aligned}
$$

Now, we compute $\left\langle\left(\frac{1-\lambda}{e^{t}-\lambda}\right)^{r} L i_{k}\left(1-e^{-t}\right) \mid x^{n+1}\right\rangle$ in two different ways. 
On the one hand,

$$
\begin{aligned}
& \left\langle\left(\frac{1-\lambda}{e^{t}-\lambda}\right)^{r} L i_{k}\left(1-e^{-t}\right) \mid x^{n+1}\right\rangle \\
& =\left\langle\left(\frac{1-\lambda}{e^{t}-\lambda}\right)^{r} \frac{L i_{k}\left(1-e^{-t}\right)}{1-e^{-t}} \mid\left(1-e^{-t}\right) x^{n+1}\right\rangle \\
& =\left\langle\left(\frac{1-\lambda}{e^{t}-\lambda}\right)^{r} \frac{L i_{k}\left(1-e^{-t}\right)}{1-e^{-t}} \mid x^{n+1}-(x-1)^{n+1}\right\rangle \\
& =\sum_{m=0}^{n}\left(\begin{array}{c}
n+1 \\
m
\end{array}\right)(-1)^{n-m}\left\langle\left(\frac{1-\lambda}{e^{t}-\lambda}\right)^{r} \frac{L i_{k}\left(1-e^{-t}\right)}{1-e^{-t}} \mid x^{m}\right\rangle \\
& =\sum_{m=0}^{n}\left(\begin{array}{c}
n+1 \\
m
\end{array}\right)(-1)^{n-m}\left\langle 1 \mid T_{m}^{(r, k)}(x \mid \lambda)\right\rangle \\
& =\sum_{m=0}^{n}\left(\begin{array}{c}
n+1 \\
m
\end{array}\right)(-1)^{n-m} T_{m}^{(r, k)}(\lambda) .
\end{aligned}
$$

On the other hand, we get

$$
\begin{aligned}
& \left\langle\left(\frac{1-\lambda}{e^{t}-\lambda}\right)^{r} L i_{k}\left(1-e^{-t}\right) \mid x^{n+1}\right\rangle \\
& =\left\langle L i_{k}\left(1-e^{-t}\right) \mid\left(\frac{1-\lambda}{e^{t}-\lambda}\right)^{r} x^{n+1}\right\rangle \\
& =\left\langle\int_{0}^{t}\left(L i_{k}\left(1-e^{-s}\right)\right)^{\prime} d s \mid H_{n+1}^{(r)}(x \mid \lambda)\right\rangle \\
& =\left\langle\int_{0}^{t} e^{-s} \frac{L i_{k}\left(1-e^{-s}\right)}{\left(1-e^{-s}\right)} d s \mid H_{n+1}^{(r)}(x \mid \lambda)\right\rangle \\
& =\sum_{l=0}^{n}\left(\sum_{m=0}^{l}\left(\begin{array}{c}
l \\
m
\end{array}\right)(-1)^{l-m} B_{m}^{(k-1)}\right) \frac{1}{l !}\left\langle\int_{0}^{t} s^{l} d s \mid H_{n+1}^{(r)}(x \mid \lambda)\right\rangle \\
& =\sum_{l=0}^{n} \sum_{m=0}^{l}\left(\begin{array}{c}
l \\
m
\end{array}\right)(-1)^{l-m} \frac{B_{m}^{(k-1)}}{(l+1) !}\left\langle t^{l+1} \mid H_{n+1}^{(r)}(x \mid \lambda)\right\rangle \\
& =\sum_{l=0}^{n} \sum_{m=0}^{l}\left(\begin{array}{c}
l \\
m
\end{array}\right)\left(\begin{array}{c}
n+1 \\
l+1
\end{array}\right)(-1)^{l-m} B_{m}^{(k-1)} H_{n-l}^{(r)}(\lambda) .
\end{aligned}
$$

Therefore, by (2.23) and (2.24), we obtain the following theorem.

Theorem 2.6 For $r, k \in \mathbb{Z}, n \in \mathbb{Z}_{\geq 0}$, we have

$$
\begin{aligned}
& \sum_{m=0}^{n}\left(\begin{array}{c}
n+1 \\
m
\end{array}\right)(-1)^{n-m} T_{m}^{(r, k)}(\lambda) \\
& \quad=\sum_{l=0}^{n} \sum_{m=0}^{l}(-1)^{l-m}\left(\begin{array}{c}
l \\
m
\end{array}\right)\left(\begin{array}{c}
n+1 \\
l+1
\end{array}\right) B_{m}^{(k-1)} H_{n-l}^{(r)}(\lambda) .
\end{aligned}
$$


Now, we consider the following two Sheffer sequences:

$$
\begin{aligned}
& T_{n}^{(r, k)}(x \mid \lambda) \sim\left(\left(\frac{e^{t}-\lambda}{1-\lambda}\right)^{r} \frac{1-e^{-t}}{L i_{k}\left(1-e^{-t}\right)}, t\right), \\
& \mathbb{B}^{(s)} \sim\left(\left(\frac{e^{t}-1}{t}\right)^{s}, t\right),
\end{aligned}
$$

where $s \in \mathbb{Z}_{\geq 0}, r, k \in \mathbb{Z}$ and $\lambda \in \mathbb{C}$ with $\lambda \neq 1$. Let us assume that

$$
T_{n}^{(r, k)}(x \mid \lambda)=\sum_{m=0}^{n} C_{n \cdot m} \mathbb{B}_{m}^{(s)}(x)
$$

By (1.21) and (2.26), we get

$$
\begin{aligned}
C_{n, m} & =\frac{1}{m !}\left\langle\left(\frac{e^{t}-1}{t}\right)^{s}\left(\frac{1-\lambda}{e^{t}-\lambda}\right)^{r} \frac{L i_{k}\left(1-e^{-t}\right)}{1-e^{-t}} t^{m} \mid x^{n}\right\rangle \\
& =\frac{1}{m !}\left\langle\left(\frac{e^{t}-1}{t}\right)^{s}\left(\frac{1-\lambda}{e^{t}-\lambda}\right)^{r} \frac{L i_{k}\left(1-e^{-t}\right)}{1-e^{-t}} \mid t^{m} x^{n}\right\rangle \\
& =\left(\begin{array}{c}
n \\
m
\end{array}\right)\left\langle\left(\frac{e^{t}-1}{t}\right)^{s}\left(\frac{1-\lambda}{e^{t}-\lambda}\right)^{r} \frac{L i_{k}\left(1-e^{-t}\right)}{1-e^{-t}} \mid x^{n-m}\right\rangle \\
& =\left(\begin{array}{c}
n \\
m
\end{array}\right) \sum_{l=0}^{n-m} \frac{s !}{(l+s) !} S_{2}(l+s, s)\left\langle\left(\frac{1-\lambda}{e^{t}-\lambda}\right)^{r} \frac{L i_{k}\left(1-e^{-t}\right)}{1-e^{-t}} \mid t^{l} x^{n-m}\right\rangle \\
& =\left(\begin{array}{c}
n \\
m
\end{array}\right) \sum_{l=0}^{n-m} \frac{s ! l !}{(l+s) !} \frac{(n-m)_{l}}{l !} S_{2}(l+s, s)\left\langle 1 \mid T_{n-m-l}^{(r, k)}(x \mid \lambda)\right\rangle \\
& =\left(\begin{array}{c}
n \\
m
\end{array}\right) \sum_{l=0}^{n-m} \frac{\left(\begin{array}{c}
n-m \\
l
\end{array}\right)}{\left(\begin{array}{c}
s+l \\
l
\end{array}\right)} S_{2}(l+s, s) T_{n-m-l}^{(r, k)}(\lambda) .
\end{aligned}
$$

Therefore, by (2.26) and (2.27), we obtain the following theorem.

Theorem 2.7 For $r, k \in \mathbb{Z}, s \in \mathbb{Z}_{\geq 0}$, we have

$$
T_{n}^{(r, k)}(x \mid \lambda)=\sum_{m=0}^{n}\left\{\left(\begin{array}{c}
n \\
m
\end{array}\right) \sum_{l=0}^{n-m} \frac{\left(\begin{array}{c}
n-m \\
l
\end{array}\right)}{\left(\begin{array}{c}
s+l \\
l
\end{array}\right)} S_{2}(l+s, s) T_{n-m-l}^{(r, k)}(\lambda)\right\} \mathbb{B}_{m}^{(s)}(x) .
$$

From (1.3) and (2.1), we note that

$$
\begin{aligned}
& T_{n}^{(r, k)}(x \mid \lambda) \sim\left(\left(\frac{e^{t}-\lambda}{1-\lambda}\right)^{r} \frac{1-e^{-t}}{L i_{k}\left(1-e^{-t}\right)}, t\right), \\
& E_{n}^{(r, s)}(x) \sim\left(\left(\frac{e^{t}+1}{2}\right)^{s}, t\right),
\end{aligned}
$$

where $r, k \in \mathbb{Z}, s \in \mathbb{Z}_{\geq 0}$.

By the same method, we get

$$
T_{n}^{(r, k)}(x \mid \lambda)=\frac{1}{2^{s}} \sum_{m=0}^{n}\left\{\left(\begin{array}{l}
n \\
m
\end{array}\right) \sum_{j=0}^{s}\left(\begin{array}{l}
s \\
j
\end{array}\right) T_{n-m}^{(r, k)}(j)\right\} E_{m}^{(s)}(x) .
$$


From (1.1) and (2.1), we note that

$$
\begin{aligned}
& T_{n}^{(r, k)}(x \mid \lambda) \sim\left(\left(\frac{e^{t}-\lambda}{1-\lambda}\right)^{r} \frac{1-e^{-t}}{L i_{k}\left(1-e^{-t}\right)}, t\right), \\
& H_{n}^{(s)}(x \mid \mu) \sim\left(\left(\frac{e^{t}-\mu}{1-\mu}\right)^{s}, t\right),
\end{aligned}
$$

where $r, k \in \mathbb{Z}$, and $\lambda, \mu \in \mathbb{C}$ with $\lambda \neq 1, \mu \neq 1, s \in \mathbb{Z}_{\geq 0}$.

Let us assume that

$$
T_{n}^{(r, k)}(x \mid \lambda)=\sum_{m=0}^{n} C_{n, m} H_{m}^{(s)}(x \mid \mu)
$$

By (1.21) and (2.31), we get

$$
\begin{aligned}
C_{n, m} & =\frac{1}{m !}\left\langle\left(\frac{e^{t}-\mu}{1-\mu}\right)^{s}\left(\frac{1-\lambda}{e^{t}-\lambda}\right)^{r} \frac{L i_{k}\left(1-e^{-t}\right)}{1-e^{-t}} t^{m} \mid x^{n}\right\rangle \\
& =\frac{\left(\begin{array}{l}
n \\
m
\end{array}\right)}{(1-\mu)^{s}}\left\langle\left(e^{t}-\mu\right)^{s} \mid\left(\frac{1-\lambda}{e^{t}-\lambda}\right)^{r} \frac{L i_{k}\left(1-e^{-t}\right)}{1-e^{-t}} x^{n-m}\right\rangle \\
& =\frac{\left(\begin{array}{c}
n \\
m
\end{array}\right)}{(1-\mu)^{s}} \sum_{j=0}^{s}\left(\begin{array}{l}
s \\
j
\end{array}\right)(-\mu)^{s-j}\left\langle e^{j t} \mid T_{n-m}^{(r, k)}(x \mid \lambda)\right\rangle \\
& =\frac{\left(\begin{array}{l}
n \\
m
\end{array}\right)}{(1-\mu)^{s}} \sum_{j=0}^{s}\left(\begin{array}{l}
s \\
j
\end{array}\right)(-\mu)^{s-j} T_{n-m}^{(r, k)}(j \mid \lambda) .
\end{aligned}
$$

Therefore, by (2.31) and (2.32), we obtain the following theorem.

Theorem 2.8 For $r, k \in \mathbb{Z}, s \in \mathbb{Z}_{\geq 0}$, we have

$$
T_{n}^{(r, k)}(x \mid \lambda)=\frac{1}{(1-\mu)^{s}} \sum_{m=0}^{n}\left\{\left(\begin{array}{l}
n \\
m
\end{array}\right) \sum_{j=0}^{s}\left(\begin{array}{l}
s \\
j
\end{array}\right)(-\mu)^{s-j} T_{n-m}^{(r, k)}(j \mid \lambda)\right\} H_{m}^{(s)}(x \mid \mu) .
$$

It is known that

$$
\begin{aligned}
& T_{n}^{(r, k)}(x \mid \lambda) \sim\left(\left(\frac{e^{t}-\lambda}{1-\lambda}\right)^{r} \frac{1-e^{-t}}{L i_{k}\left(1-e^{-t}\right)}, t\right), \\
& (x)_{n} \sim\left(1, e^{t}-1\right) .
\end{aligned}
$$

Let

$$
T_{n}^{(r, k)}(x \mid \lambda)=\sum_{m=0}^{n} C_{n, m}(x)_{m}
$$

Then, by (1.21) and (2.34), we get

$$
\begin{aligned}
C_{n, m} & =\frac{1}{m !}\left\langle\left(\frac{1-\lambda}{e^{t}-\lambda}\right)^{r} \frac{L i_{k}\left(1-e^{-t}\right)}{1-e^{-t}}\left(e^{t}-1\right)^{m} \mid x^{n}\right\rangle \\
& =\sum_{l=0}^{\infty} \frac{S_{2}(l+m, m)}{(l+m) !}\left\langle\left(\frac{1-\lambda}{e^{t}-\lambda}\right)^{r} \frac{L i_{k}\left(1-e^{-t}\right)}{1-e^{-t}} \mid t^{m+l} x^{n}\right\rangle
\end{aligned}
$$




$$
\begin{aligned}
& =\sum_{l=0}^{n-m} \frac{S_{2}(l+m, m)}{(l+m) !}(n)_{m+l}\left\langle 1 \mid\left(\frac{1-\lambda}{e^{t}-\lambda}\right)^{r} \frac{L i_{k}\left(1-e^{-t}\right)}{1-e^{-t}} x^{n-m-l}\right\rangle \\
& =\sum_{l=0}^{n-m}\left(\begin{array}{c}
n \\
l+m
\end{array}\right) S_{2}(l+m, m) T_{n-m-l}^{(r, k)}(\lambda) .
\end{aligned}
$$

Therefore, by (2.34) and (2.35), we obtain the following theorem.

Theorem 2.9 For $r, k \in \mathbb{Z}$, we have

$$
T_{n}^{(r, k)}(x \mid \lambda)=\sum_{m=0}^{n}\left\{\sum_{l=0}^{n-m}\left(\begin{array}{c}
n \\
l+m
\end{array}\right) S_{2}(l+m, m) T_{n-m-l}^{(r, k)}(\lambda)\right\}(x)_{m} .
$$

Finally, we consider the following two Sheffer sequences:

$$
\begin{aligned}
& T_{n}^{(r, k)}(x \mid \lambda) \sim\left(\left(\frac{e^{t}-\lambda}{1-\lambda}\right)^{r} \frac{1-e^{-t}}{L i_{k}\left(1-e^{-t}\right)}, t\right), \\
& x^{[n]} \sim\left(1,1-e^{-t}\right),
\end{aligned}
$$

where $x^{[n]}=x(x+1) \cdots(x+n-1)$.

Let us assume that

$$
T_{n}^{(r, k)}(x \mid \lambda)=\sum_{m=0}^{n} C_{n, m} x^{[m]}
$$

Then, by (1.21) and (2.37), we get

$$
\begin{aligned}
C_{n, m} & =\frac{1}{m !}\left\langle\left(\frac{1-\lambda}{e^{t}-\lambda}\right)^{r} \frac{L i_{k}\left(1-e^{-t}\right)}{1-e^{-t}}\left(1-e^{-t}\right)^{m} \mid x^{n}\right\rangle \\
& =\sum_{l=0}^{\infty} \frac{(-1)^{l} S_{2}(l+m, m)}{(l+m) !}\left\langle\left(\frac{1-\lambda}{e^{t}-\lambda}\right)^{r} \frac{L i_{k}\left(1-e^{-t}\right)}{1-e^{-t}} \mid t^{m+l} x^{n}\right\rangle \\
& =\sum_{l=0}^{n-m} \frac{(-1)^{l} S_{2}(l+m, m)}{(l+m) !}(n)_{m+l}\left\langle 1 \mid\left(\frac{1-\lambda}{e^{t}-\lambda}\right)^{r} \frac{L i_{k}\left(1-e^{-t}\right)}{1-e^{-t}} x^{n-m-l}\right\rangle \\
& =\sum_{l=0}^{n-m}(-1)^{l}\left(\begin{array}{c}
n \\
l+m
\end{array}\right) S_{2}(l+m, m) T_{n-m-l}^{(r, k)}(\lambda) .
\end{aligned}
$$

Therefore, by (2.37) and (2.38), we obtain the following theorem.

Theorem 2.10 For $r, k \in \mathbb{Z}, n \geq 0$, we have

$$
T_{n}^{(r, k)}(x \mid \lambda)=\sum_{m=0}^{n}\left\{\sum_{l=0}^{n-m}(-1)^{l}\left(\begin{array}{c}
n \\
l+m
\end{array}\right) S_{2}(l+m, m) T_{n-m-l}^{(r, k)}(\lambda)\right\} x^{[m]} .
$$


Authors' contributions

All authors contributed equally to the manuscript and typed, read, and approved the final manuscript.

\section{Author details}

'Department of Mathematics, Sogang University, Seoul, 121-742, Republic of Korea. ${ }^{2}$ Department of Mathematics, Kwangwoon University, Seoul, 139-701, Republic of Korea.

\section{Acknowledgements}

This work was supported by the National Research Foundation of Korea (NRF) grant, funded by the Korea government (MOE) (No. 2012R1A1A2003786).

Received: 11 July 2013 Accepted: 6 August 2013 Published: 20 August 2013

\section{References}

1. Araci, S, Acikgoz, M: A note on the Frobenius-Euler numbers and polynomials associated with Bernstein polynomials. Adv. Stud. Contemp. Math. 22(3), 399-406 (2012)

2. Kim, T: An identity of the symmetry for the Frobenius-Euler polynomials associated with the fermionic $p$-adic invariant $q$-integrals on $\mathbb{Z}_{p}$. Rocky Mt. J. Math. 41(1), 239-247 (2011)

3. Kim, T: Identities involving Frobenius-Euler polynomials arising from non-linear differential equations. J. Number Theory 132(1), 2854-2865 (2012)

4. Ryoo, C: A note on the Frobenius-Euler polynomials. Proc. Jangjeon Math. Soc. 14(4), 495-501 (2011)

5. Ryoo, CS, Agarwal, RP: Exploring the multiple Changhee q-Bernoulli polynomials. Int. J. Comput. Math. 82(4), 483-493 (2005)

6. Kim, DS, Kim, T, Kim, YH, Lee, SH: Some arithmetic properties of Bernoulli and Euler numbers. Adv. Stud. Contemp. Math. 22(4), 467-480 (2012)

7. Kim, DS, Kim, T: Poly-Bernoulli polynomials arising from umbral calculus (communicated)

8. Kim, T: Power series and asymptotic series associated with the $q$-analog of the two-variable $p$-adic $L$-function. Russ. J. Math. Phys. 12(2), 186-196 (2005)

9. Can, M, Cenkci, M, Kurt, V, Simsek, Y: Twisted Dedekind type sums associated with Barnes' type multiple Frobenius-Euler /-functions. Adv. Stud. Contemp. Math. 18(2), 135-160 (2009)

10. Ding, D, Yang, J: Some identities related to the Apostol-Euler and Apostol-Bernoulli polynomials. Adv. Stud. Contemp. Math. 20(1), 7-21 (2010)

11. Kim, T, Choi, J: A note on the product of Frobenius-Euler polynomials arising from the $p$-adic integral on $\mathbb{Z}_{p}$. Adv. Stud. Contemp. Math. 22(2), 215-223 (2012)

12. Kurt, B, Simsek, Y: On the generalized Apostol-type Frobenius-Euler polynomials. Adv. Differ. Equ. 2013, 1 (2013)

13. Simsek, Y, Yurekli, O, Kurt, V: On interpolation functions of the twisted generalized Frobenius-Euler numbers. Adv. Stud. Contemp. Math. 15(2), 187-194 (2007)

14. Roman, S: The Umbral Calculus. Pure and Applied Mathematics, vol. 111. Academic Press, New York (1984)

15. Roman, S, Rota, G-C: The umbral calculus. Adv. Math. 27(2), 95-188 (1978)

doi:10.1186/1687-1847-2013-25

Cite this article as: Kim and Kim: Higher-order Frobenius-Euler and poly-Bernoulli mixed-type polynomials. Advances in Difference Equations 2013 2013:251.

\section{Submit your manuscript to a SpringerOpen ${ }^{\ominus}$ journal and benefit from:}

- Convenient online submission

- Rigorous peer review

Immediate publication on acceptance

- Open access: articles freely available online

- High visibility within the field

- Retaining the copyright to your article 